\title{
Implementation and Evaluation of Integrity Protection Facilities for Active Grids
}

\author{
Arso Savanović ${ }^{1}$, Dušan Gabrijelčič ${ }^{1}$, Borka Jerman Blažič ${ }^{1}$, and Janez Bešter ${ }^{2}$ \\ 1 Jožef Stefan Institute, Laboratory for Open Systems and Networks, \\ Jamova 39, 1000 Ljubljana, Slovenia, \\ 2 University of Ljubljana, Laboratory for Telecommunications, \\ Tržaška 25, 1000 Ljubljana, Slovenia
}

\begin{abstract}
Some recent studies have shown that Grid systems can benefit from active networks technologies, thus a novel networking paradigm called Active Grid has been proposed. On the other hand, the development of appropriate security components for Grid systems is still in an early stage due to complexity and mostly academic use of Grids to date. However, security issues are exacerbated in Active Grids due to the availability of processing and caching capacity in intermediate network nodes, which adds to Grid complexity. In this paper we describe a set of facilities for protection of data integrity in Active Grids, and then present and discuss some experimental results from validation and evaluation test with the prototype implementation of these facilities.
\end{abstract}

\section{Introduction}

Some recent studies have shown that Grid systems can benefit from active networks technologies [1215[13, thus a novel networking paradigm called Active Grid has been proposed. The main idea is to place active network nodes (ANNs) at strategic points in the network and to make processing and caching capabilities of ANNs explicitly available to Grid applications. Examples of such Active Grid configurations include Meta-cluster computing, where an ANNs is used as a network head of each cluster or parallel computer, and Global computing, where an ANN is used to manage a single Grid node or a collection of Grid nodes [12].

On the other hand, the development of appropriate security components for Grid systems is still in an early stage, which is largely due to Grid complexity and mostly academic use of Grids to date. Existing Grid toolkits do provide some security features, e.g. 872, however many important challenges still lie ahead, including but not limited to the Grid security requirements listed in [17. These open issues are even more challenging in Active Grids due to the availability of processing and caching capacity in intermediate network nodes, which adds to the already recognised complexity of Grids. In this paper we focus on one aspect of security, the integrity service, and present a set of facilities for integrity protection, which can be applied to Active Grids. These facilities are a part of a comprehensive security architecture for active networks based systems [19, which has been developed, implemented and tested within the FAIN project [6] from the EU VI. Framework Programme. 


\section{On Integrity in Active Grids}

An integrity service protects against unauthorised changes of data while in transit over the network, including both intentional change or destruction and accidental change or loss. Unauthorised changes include modifications, replay, and spoofing of data.

With the growing industry support an important tendency in Grid development is gradual commercialisation of Grids, which increases the incentive for unauthorised change of data, since this kind of mischief generates direct economic benefits for the adversary. For illustration consider the following example. A provider of a commercial computational Grid offers processing capacity to its customers in return for a financial compensation. Assume that the use of Grid is based on prepaid digital tokens. Obviously, there is an incentive for Grid users to tamper with these tokens in order to reduce or even eliminate the costs related to the use of commercial Grid resources. Without integrity protection facilities in place, a skillful adversary would be able to cheat in different ways, e.g. : (a) buy a single token, but use it several times by replaying it to the Grid accounting system, (b) change the nominal value of the token to allow the use of more resources than what was originally paid for, or (c) sniff the network and intercept someone else's tokens, then change the owner ID in these tokens, effectively stealing them.

Traditionally, integrity has been provided on an end-to-end basis, which is a valid approach for current Grid systems, where Grid nodes are mapped to end nodes and Grid application data is processed only at these end nodes. However, the availability of ANNs in Active Grids makes it possible to process application data at intermediate network nodes as well. Thus, Grid application data can legally change while in transit over the network, which requires a different approach to integrity protection. In fact, this requirement is not specific to Active Grids but is valid in general for systems based on active networks technologies.

\section{Integrity Protection Facilities for Active Grids}

Even though Active Grids offer Grid applications processing capabilities at intermediate network nodes, not all Grid application data require processing in these nodes, i.e. Grid application data can be divided into two classes: static (end-toend) data and dynamic data. Consequently, our integrity protection service is based on a combination of end-to-end protection and hop-by-hop protection.

Both types of protection are based on a specific packet format, which has to provide for different treatment of static and dynamic Grid application data. We have adopted the ANEP, Active Networks Encapsulation Protocol [3], because of its extensibility and because the majority of active network approaches support it. In the context of ANEP we have defined several options to support the FAIN security architecture. These options carry a service identifier (eg. label), hop protection and credentials option related information, service variable data and resource usage information. Integrity protection is based on the hop protection, credential and variable data options. 


\subsection{End-to-End Protection}

The end-to-end protection is facilitated through the use of the ANEP credential option. The credential option is generated at the source and is never modified. Every receiver of the ANEP packet can verify the integrity of static data in the ANEP packet by processing this option. The credential option is defined by the credential identifier and type, location field (specifying where the credentials can be fetched), target field (where the user can specify specific targets as nodes, system layer or a packet itself), optional timestamp (protects against replays), and the digital signature. The digital signature covers only static data within an ANEP packet, which includes the first 32 bits of basic ANEP header, source address, service identifier, ANEP payload, and the credential option itself except digital signature data. There can be zero, one, or more credential options in a single ANEP packet. On each visited ANN the relevant credentials are fetched, the certification path is validated, and the digital signature is verified. We note that the digital signature mechanism provides authentication of data origin and nonrepudiation security services in addition to the end-to-end integrity protection.

Various credential types are supported: X.509 certificates, attribute certificates [1], SPKI certificates or Keynote credentials [5]. Unless included in the packet, the credentials can be fetched from a suitable store, e.g. DNS or LDAP. In our case, we have designed and implemented a simple protocol that enables fetching credentials from the previous hop ANN. In this way, the originating entity is responsible for supplying all the credentials, which may be required later on the nodes that a packet traverses. To be able to supply credentials on the intermediate nodes we have designed and implemented node credentials cache. After successful validation, the credentials are cached on the node for the time of their validity or according to the cache policy on cache size and maximum time period of a cache entry. Caching credential has also other benefits: if the cache entry is valid, there is no need to validate the credentials. In this way we can reduce required digital signature validation to only one per credential option in the packet, which results in a significant speed improvement, after the first principal packet has passed the node. Additionally, we cache bad credentials in a separate cache in the cases, when the credentials cannot be verified. Subsequent packets with bad credentials are discarded immediately.

\subsection{Hop-by-Hop Protection}

While the ANEP data option serves as a container for transporting dynamic Active Grid application data, the hop-by-hop integrity protection is facilitated with the ANEP hop protection option. Each ANN in an Active Grid maintains a (separate) shared secret key with each of its neighbour ANNs. When an ANN receives an ANEP packet, it a) uses the secret key it shares with the previous hop ANN to process the hop protection option and thus check the integrity of dynamic data in the ANEP packet, and b) uses the secret key it shares with the next hop ANN to generate a new hop protection option before forwarding the ANEP packet towards its destination. 
The hop protection option is defined by a triple of Security Association (SA) identifier, which points to the respective association with a neighbour node, sequence number field, which protects against replays, and keyed hash. The keyed hash covers the entire ANEP packet except the keyed hash itself. The per-hop protection protects all ANEP packets exchanged between two neighbour ANNs. When the packet leaves the node a new hop protection option is built based on the respective SA for the next hop ANN.

Dynamic and scalable hop protection requires at least two supporting protocols. First, a SA setup protocol that supports setup and maintenance of SA (including key exchange) between two neighbour ANNs, and second, a neighbour discovery protocol. For the former we have adopted the Station-to-Station protocol 20], additionally protected with end-to-end protection, as described in section 3.1 Neighbour discovery is described in the next section.

\subsection{Neighbour Discovery}

As seen above, the hop-by-hop integrity protection requires each ANN in an Active Grid to keep an up-to-date list of all its neighbour ANNs. Manual configuration of ANN neighbour lists is not acceptable due to scalability issues and dynamic nature of Active Grids. We have therefore developed a protocol, which enables ANNs in an Active Grid to automatically create a list of neighbour ANNs and update it whenever necessary due to changes in the network infrastructure [18.

The protocol has three operation modes: node advertisements, discovery handshake, and heartbeat exchange. ANNs advertise themselves by periodically transmitting the advertisement message to an emulated communications bus, which distributes the advertisement to all ANNs. The implementation of an emulated communications bus can be multicast-based, with one dedicated multicast group per overlay, server-based, or a combination of both. Upon reception of an advertisement an ANN starts a discovery handshake with the sender with probability $p$ only in order to limit the load on the sender of the advertisement. The discovery handshake is the main protocol mechanism for neighbour discovery and detection of network changes. It involves an exchange of two messages between the node, which initiates the handshake (poller), and the node, which responds (responder). Both messages carry the address of the previous hop ANN, which is used by ANNs en route for updating their neighbour tables when necessary. A node periodically sends the heartbeat message to each of its known neighbours to indicate that it is still alive. Neighbour ANNs use this message to refresh the soft state in their neighbour tables. We note that neighbour discovery protocol employs packet interception, where an intermediate ANN intercepts and processes the protocol message, even though the message is addressed to some other node. Packet interception is inherently supported by ANNs [21] and is an example of how Grid systems can benefit from active network technologies. 


\section{Proof-of-Concept Implementation and Experimental Results}

The proof-of-concept implementation of the facilities described above has been developed in order to verify end evaluate the operation of the integrity service provided by these facilities. The facilities have been tested in a series of lab tests and demonstrated at the FAIN project Final Review and Demonstration Event.

The testing environment was set up on a commodity PC, with Intel P4 $2.2 \mathrm{GHz}$ processor, $512 \mathrm{MB}$ RAM, Red Hat Linux 8.0, kernel 2.4.18-14, Java SDK 1.3.1_3, the Bouncy Castle crypto-library version 1.17, and the FAIN ANN code. In our tests ANEP packets carried basic ANEP header, a hop protection option, a service identifier option, a single credential option, a resource option and a zero length variable option and payload. The keyed hash algorithm used in the hop protection option was HMAC-SHA-1 9]. The credential used was an X.509 [1] based certificate signed with a combination of RSA encryption and MD5 hash. The signature in the credential option, used for end-to-end protection, was an RSA encrypted SHA-1 hash. The RSA key length was 768 bits and the certification path length was one.

Figure 1 shows the breakdown of the measured security costs related to an ANEP packet traversing an ANN. The "hop" part represents the costs of processing the hop protection option, i.e. validating the option on the receiving side and building a new option on the sending side. The "encoding/decoding" part represents the costs of parsing a packet at the receiving side and forming a new packet at the sending side. The "other costs" part represents the costs of ANN building a security context of the packet. The "signature" part refers to the costs of validating a credential and verifying the digital signature in the packet.

The left hand side of the figure depicts a case, when the user credential (certificate) is contained in the ANEP packet (but not yet verified), while the right hand side of the figure depicts a case, when the user credential is already in the cache. As expected, the largest overhead is due to the credential option processing, since this involves the costly asymmetric cryptography (RSA signatures validation). With no caching of credentials, these costs represent as much as $85 \%$ of all security related costs, while the hop-by-hop protection related costs represent around $8 \%$. When credentials are cached (from previous packets), the digital signature related costs are reduced to $50 \%$. The hop-by-hop protection related costs remain the same, however their percentage raises to $25 \%$ due to a reduction of overall costs.

In absolute numbers, we were able to reach the ANN throughput of 396 packets per second without the credential cache. The use of the credential cache yields the threefold improvement, i.e. an ANN is capable of handling 1190 packets per second. When only hop-by-hop protection is activated, the ANN can handle more than 7000 packets per second. Our measurements show that security related costs are not drastically related to the RSA key size in current setup: in the case of RSA512 the node can pass 1340 and in case of RSA1024 1003 packets per second.

We have also looked into the ways of reducing the performance overhead induced by our protection facilities. A comparison of the Bouncy castle crypto- 
Packet costs (Credential validation, RSA768)

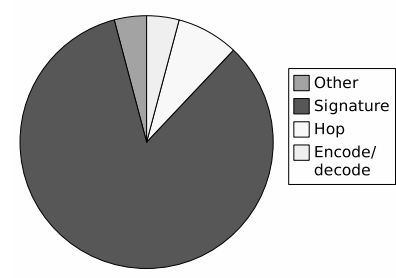

Packet costs (Cached credentials, RSA768)

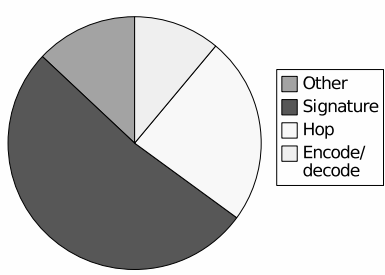

Fig. 1. Security related packet processing costs

graphic library with the OpenSSL library 1 shows that the OpenSSL outperforms the Bouncy castle by the factor of two for digital signature verification (1428 vs. 3550 verifications per sec). Cryptographic accelerators like Broadcom based BCM5821 cryptographic accelerator 2 show additional speed improvements with very light utilisation of the main CPU (8142 signatures verification per sec, less then $1 \% \mathrm{CPU}$ ). Thus, integrating native libraries or accelerators should considerably improve performance of protection facilities presented in this paper and enable ANNs to handle few thousands ANEP packets per second even with the costly checking of digital signatures.

In comparison, the existing performance results for active networks based systems are for simpler packet structures as in our case, and range from 6000 to 11000 packets per second in a similar environment, e.g ANTS [23] and Bees [22]. As far as authors are aware, so far no security nor cryptography related costs were calculated or reported for active networks based systems.

With regards to the neighbour discovery, we were mostly interested in the speed of neighbour discovery, i.e. the delay between the network change and the corresponding updates of neighbour ANN tables. By varying different protocol parameters in our tests we were able to reach convergence delay in the range of 30-40 seconds, i.e. our target values. We chose this target value because the convergence delay of current routing protocol implementations ranges from around 30s for OSPF 4 to several minutes for BGP [11]. We find the comparison with the routing protocols interesting due to the following. As far as the authors are aware, no results on convergence delay are currently available for related efforts in the area of automatic discovery of neighbour ANNs (e.g. [14]). On the other hand, routing protocols provide analogous functionality to neighbour discovery protocol: after a perturbation occurs in the network, the routing protocol has to accordingly reconfigure the physical network (i.e. routes in the network) as soon as possible in order to minimise the disruption in communication caused by the perturbation.

\footnotetext{
${ }^{1}$ http://www $. o p e n s s l . o r g$

${ }^{2}$ http://www.broadcom.com/products/5821.html
} 


\section{Related Work and Conclusions}

Some of the existing Grid toolkits provide support for integrity protection, see e.g. 87/2, however these protection facilities are based on an end-to-end approach, i.e. they completely preclude modifications of data while in transit over the network. This breaks the Active Grid model, which renders these integrity protection facilities unsuitable for Active Grids.

In 10] authors have presented a framework for providing hop-by-hop security in active network testbeds. This framework is heavily based on off the shelf security solutions. More importantly, the framework raises serious scalability concerns, since it is centralised and involves manual configuration of active network nodes. Thus, this work has demonstrated a more practical and short term solution for existing testbeds based on active networks technologies rather than a true long term security solution.

SANTS [16] provides a strong and relatively complete security solution for active networks based systems. Integrity protection in SANTS is also based on the separation of end-to-end and hop-by-hop concerns, and their solution is similar to ours. However, in SANTS there is no mechanisms for automatic discovery and configuration of neighbour active nodes, which is a basic prerequisite for (scalable) hop-by-hop protection. Neither do they report on performance of their prototype.

In this paper, we have presented a working example of a complete set of integrity protection facilities suited for communications systems based on active networks technologies, including the novel paradigm of Active Grids. The facilities are a part of a comprehensive security architecture, which has been designed, implemented and evaluated within the EU FAIN project. This work does not replace but rather complements the work in other Grid security efforts, e.g. within GGF.

In general, providing security is costly in performance terms, since strong security solutions employ cryptographic techniques. We believe that performance results presented in this paper contribute towards better understanding of performance limits imposed by security facilities. Based on the performance results from our evaluation tests with the prototype and existing reports on performance of some active networks systems, we find the performance of integrity protection facilities completely suitable for the control and management planes of Active Grids. In the transport plane, however, the integrity protection facilities may not be able to meet the requirements of those Active Grid applications demanding higher performance.

\section{References}

1. Information Technology - Open Systems Interconnection - The Directory: Publickey and Attribute Certificate Frameworks. International Standard, March 2000.

2. Uniform Interface to Computing Resources. UNICORE Plus Final Report, December 2002. http://www.unicore.org/. 
3. D. S. Alexander et. al. Active Network Encapsulation Protocol (ANEP). Active Network Group draft, July 1997.

4. A. Basu and J. G. Riecke. Stability Issues in OSPF Routing. In Proceedings of SIGCOMM 2001, pages 225-236, August 2001.

5. M. Blaze, J. Feigenbaum, J. Ioannidis, and A. D. Keromytis. The KeyNote TrustManagement System, Version 2. RFC 2704, September 1999.

6. FAIN-Future Active IP Networks. http://www.ist-fain.org.

7. A. Ferrari et. al. A Flexible Security System for Metacomputing Environments. Technical Report CS-98-36, University of Virginia, Charlottesville, VA 22903, USA, December 1998.

8. Grid Security Infrastructure (GSI). http://www.globus.org/security/.

9. H. Krawczyk, M. Bellare, and R. Canetti. HMAC: Keyed-Hashing for Message Authentication. RFC 2104, February 1997. Informational.

10. S. Krishnaswamy, J. B. Evans, and G. J. Minden. A Prototype Framework for Providing Hop-by-Hop Security in an Experimentaly Deployed Active Network. In Werner Bob, editor, Proceedings of DANCE 2002, pages 216-224. IEEE Computer Society, 2002. May 29-30, 2002, San Francisco, USA.

11. C. Labovitz, A. Ahuja, A. Bose, and F. Jahanian. Delayed Internet Routing Convergence. In Proceedings of SIGCOMM 2000, pages 175-187, 2000.

12. L. Lefèvre et. al. Active Networking Support for the Grid. Lecture Notes in Computer Science, 2207:16-33, 2001.

13. M. Maimour and C. Pham. An Active Reliable Multicast Framework for the Grids. In International Conference on Computational Science (2), pages 588-597, 2002.

14. S. Martin and G. Leduc. RADAR: Ring-based Adaptive Discovery of Active neighbour Routers. In Proceedings of IWAN 2002, pages 62-73, December 2002.

15. N. Mohamed. Active Networks and Their Utilization in the Computational Grid. Technical Report TR03-04-01, Engineering University of Nebraska-Lincoln, Lincoln, NE 68588-0115, April 2003.

16. S. L. Murphy, E. T. Lewis, and N. M. R. Watson. Secure Active Network Prototypes. In Werner Bob, editor, Proceedings of DANCE 2002, pages 166-181. IEEE Computer Society, 2002. May 29-30, 2002, San Francisco, USA.

17. N. Nagaratnam et. al. The Security Architecture for Open Grid Services. GGF Specification, July 2002.

18. A. Savanović and B. Jerman Blažič. A Protocol for Adaptive Autoconfiguration of Active Networks. WSEAS Transactions on Communications, 2:78-83, 2003.

19. A. Savanović, D. Gabrijelčič, B. Jerman Blažič, and S. Karnouskos. An Active Networks Security Architecture. Informatica, 26(2):211-221, 2002.

20. B. Schneier. Applied Cryptography: Protocols, Algorithms, and Source Code in C. John Wiley and Sons, Inc., second edition, 1996.

21. J. M. Smith et. al. Activating Networks: A Progress Report. IEEE Computer Magazine, pages 32-41, April 1999.

22. T. Stack, E. Eide, and J. Lepreau. Bees: A Secure, Resource-Controlled, JavaBased Execution Environment, December 2002. http://www.cs.utah.edu/flux/janos/bees.html

23. D. Wetherall, J. Guttag, and D. Tennenhouse. ANTS: Network Services Without Red Tape. IEEE Computer, pages 42-48, Apr 1999. 